"Joining insured groups: how to split the emerging profit"

\begin{tabular}{|c|c|c|}
\hline AUTHORS & \multicolumn{2}{|l|}{$\begin{array}{l}\text { Elinor Mualem } \\
\text { Abraham Zaks }\end{array}$} \\
\hline ARTICLE INFO & \multicolumn{2}{|c|}{$\begin{array}{l}\text { Elinor Mualem and Abraham Zaks (2017). Joining insured groups: how to split } \\
\text { the emerging profit. Insurance Markets and Companies, 8(1), 29-33. } \\
\text { doi:10.21511/ins.08(1).2017.03 }\end{array}$} \\
\hline DOI & \multicolumn{2}{|c|}{ http://dx.doi.org/10.21511/ins.08(1).2017.03 } \\
\hline RELEASED ON & \multicolumn{2}{|l|}{ Friday, 24 November 2017} \\
\hline RECEIVED ON & \multicolumn{2}{|l|}{ Sunday, 14 May 2017} \\
\hline \multirow[t]{2}{*}{ ACCEPTED ON } & \multicolumn{2}{|l|}{ Saturday, 30 September 2017} \\
\hline & \multicolumn{2}{|l|}{$(\mathrm{cc}) \overline{\mathrm{EY}-\mathrm{NC}}$} \\
\hline LICENSE & \multicolumn{2}{|c|}{$\begin{array}{l}\text { This work is licensed under a Creative Commons Attribution-NonCommercial } 4.0 \\
\text { International License }\end{array}$} \\
\hline JOURNAL & \multicolumn{2}{|c|}{ "Insurance Markets and Companies" } \\
\hline ISSN ONLINE & \multicolumn{2}{|l|}{$2522-9591$} \\
\hline PUBLISHER & \multicolumn{2}{|c|}{ LLC "Consulting Publishing Company "Business Perspectives" } \\
\hline FOUNDER & \multicolumn{2}{|c|}{ LLC "Consulting Publishing Company "Business Perspectives" } \\
\hline \multirow{2}{*}{ NUMBER OF REFERENCES } & & 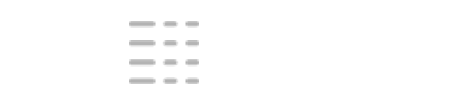 \\
\hline & NUMBER OF FIGURES & NUMBER OF TABLES \\
\hline 7 & 0 & 1 \\
\hline
\end{tabular}

(C) The author(s) 2022. This publication is an open access article. 
Elinor Mualem (Israel), Abraham Zaks (Israel)

\title{
Joining insured groups: how to split the emerging profit
}

\begin{abstract}
In the process of evaluating the premium of an insurance plan, one considers the risk arising from various uncertainties. The authors suppose for a plan whose net premium is $p$ and the standard deviation is $\sigma$ the premium including the risk factor will be $p+3 \sigma$ for a given member, and $3 \sigma$ reflects the risk. For a group of $n$ members with the same premium $p$ and with standard deviation $\sigma$, the premium including the risk factor will be $p+3 \sigma / \sqrt{n}$ where $3 \sigma / \sqrt{n}$ reflects the risk for each member of the group. The authors study the emerging profit in case of $n$ insured groups each with its own premium and its own risk when all the $n$ insured groups merge into a single group uniting all insured members. They prove that there emerge a profit due to joining the $n$ groups into a single one due to a reduced total risk of the $n$ separate insured groups when merging into a single group. The emerging profit between the various groups may be divided using the Shapley values method or using utility functions for each group. The auhors discuss various reasonable ways to split the emerging profit between the $n$ groups and show that the split of the profit depends on the chosen method. The main tools are techniques of game theory, in particular those of cooperative games.
\end{abstract}

Keywords: risk, standard deviation, Shapley value, Kahneman-Tverski utility function.

JEL Classification: C70, C71, G22.

Received on: $14^{\text {th }}$ of May, 2017.

Accepted on: $30^{\text {th }}$ of September, 2017.

\section{Introduction}

In the study of life insurance, an insured person pays a premium for a prescribed period while he is alive and gets a benefit from the insurance company when he dies or for a prescribed period provided he is alive. We consider the standard deviation as a measure for the risk from the insurance company's point of view. Suppose there are given $k$ insured groups. What are the consequences of their merging into one group from the point of view of the risk.

Let for group $i$, for $i=1, \ldots, n_{i}$ be the number of insured persons, $n_{i} p_{i}$ be the total net premium and $\sqrt{n_{i}} \sigma_{i}$ be the total standard deviation when considered as a random variable. Then, an insured person in company $I$ pays the net premium $p_{i}$ and an additional risk term, say $3 \sigma_{i} / \sqrt{n_{i}}$ so that his total premium amounts to:

$p_{i}+\frac{3 \sigma_{i}}{\sqrt{n_{i}}}$.

The total premium received from all $k$ companies is

$\sum_{i=1}^{k} p_{i}+3 \sum_{i=1}^{k} \sqrt{n_{i} \sigma_{i}^{2}}$

(c) Elinor Mualem, Abraham Zaks, 2017.

Elinor Mualem, Department of Mathematics Technion, Haifa, Israel. Abraham Zaks, Professor Emeritus, Department of Mathematics Technion, Actuarial Research Center, Haifa University, Haifa, Israel.

This is an Open Access article, distributed under the terms of the Creative Commons Attribution-NonCommercial 4.0 International license, which permits re-use, distribution, and reproduction, provided the materials aren't used for commercial purposes and the original work is properly cited.
Now, for the merged company the following holds: the number of insured persons is $n=n_{1}+\ldots+n_{k}$, the net premium is $\left(n_{1} p_{1}+\ldots+n_{k} p_{k}\right) / n$ and the standard deviation is $\sqrt{n_{1} \sigma_{1}^{2}+\ldots+n_{k} \sigma_{k}^{2}} / n$, so the total premium needed for the merged company is

$$
\sum_{i=1}^{k} p_{i}+3 \sqrt{\sum_{i=1}^{k} n_{i} \sigma_{i}^{2}}
$$

Hence, there is a difference between what is needed and what is actually received. This difference is termed the profit that emerges when companies merge and is:

$$
3 \sum_{i=1}^{k} \sqrt{n_{i} \sigma_{i}^{2}}-3 \sqrt{\sum_{i=1}^{k} n_{i} \sigma_{i}^{2}} .
$$

We ask what are good ways to split this profit among the $k$ companies. A trivial solution to the question would be to give equal parts to all companies or give parts that are proportional to the sizes of the companies. Both these solutions are actuarially imbalanced (for example, the first solution is always better for the smaller company regardless how "risky" it is). Hence, we don't consider these as good answers.

\section{Game theory preliminaries}

We formulate the problem and solutions using tools from game theory. Based on Weber (1994), we briefly go over some important definitions.

Let $N$ be a finite set that is called the set of players. In our context, we take $N$ to be the set $\{1,2, \ldots, \mathrm{k}\}$. A coalition is a subset of $N$. The set of all coalitions is denoted by $\mathrm{P}(N)$. A cooperative game is a couple 
$(N, v)$ where $v$ is a function that assign each nonempty coalition a real number and zero to the empty coalition.

An imputation $x=\left(x_{1}, \ldots, x_{k}\right)$ is a real vector such that $x_{1} \ldots+x_{k}=v(N)$.

A stable imputation is an imputation where for each coalition $S, \sum_{i \in S} x_{i} \geq v(S)$ holds.

The set of all stable imputations is the core of the game and is denoted by $C(N, v)$. Generally speaking, an element of the core is regarded as "good" imputation (or at least satisfactory). Alas, answering if a general game has non-empty core is not a trivial task. However, for the class of convex games (to be defined shortly) the core is never empty by a theorem of Shapley, which we give next. A game is convex if for every player $i$ and for every two coalitions $S \subseteq T \subseteq N \backslash\{i\}$, the following holds:

$$
v(T \quad\{i\})-v(T) \geq v(S \quad\{i\})-v(S)
$$

The Shapley value of a game, denote by $\varphi(N, v)$ is defined as follows. Let $L$ be a linear order on $N$ and denote by $L(i)$ the set of elements in $N$ that precedes $i$ in the order $L$. We define a ${ }^{L}(N, v)$ to be the vector for which the $i$-th entry is defined to be

$v(L(i) \quad\{i\})-v(L(i))$.

Then, $\varphi(N, v)$ is defined as the average of all vectors $\mathrm{a}^{L}(N, v)$ over all $k$ ! linear orders on $N$. Namely,

$$
\varphi(N, v)=\frac{1}{k !} \sum_{L} a^{L}(N, v) .
$$

Next theorem by Shapley (1929) characterizes the cores of convex games:

Theorem (2.2). Let $(N, v)$ be a convex game. Then the core of $(N, v)$ is the convex hull of all the vectors $\mathrm{a}^{L}(N, v)$. Specifically, $\varphi(N, v)$ is in the core.

\section{Stable split}

In this section, we start to characterize an answer to the question from the first section. We fix the notions of first section. Let $M$ be some fixed amount of money to be divided among the $k$ companies. A split of $M$ is a real vector $\left(x_{1}, \ldots, x_{k}\right)$ such that the entries $x_{i}$ are nonnegative and their sum is $M$ (that is, $x_{i}$ presents the amount company $i$ gets in the split). Naturally, we take $M$ to be the amount equation (1). Let $S$ be a subset of the companies. We denote by $M_{S}$ the profit that the companies inside $S$ can generate if they merge without the companies outside $S$. That is,

$$
M_{S}=3 \sum_{i \in S} \sqrt{n_{i} \sigma_{i}^{2}}-3 \sqrt{\sum_{i \in S} n_{i} \sigma_{i}^{2}} .
$$

Note that if $S$ is the set of all companies then $M_{S}$ is the money we want to split. We define a stable split to be a split $\left(x_{1}, \ldots, x_{k}\right)$ for which

$$
\forall S \subseteq\{1, \quad, k\} \quad \sum_{i \in S} x_{i} \geq M_{S} .
$$

The term "stable" denotes the fact that given a stable split no company can get more money by not joining the rest of the companies. Next, we show that stable splits exist.

We define a game $(N, v)$ as follows. $N$ is the set of companies and the function $v$ is defined on nonempty subsets $S$ of $N$ to be $M_{S}$. In this context, a split is an imputation with additional requirement on non-negativity of the entries (which will be satisfied easily). For singletons $S$ (i.e., containing single company), we have that $M_{S}$ is zero, hence, the elements in the core of the game are exactly all the stable splits. We show that the core is not empty and give explicit vector there by showing that $(N, v)$ is convex and then using theorem (2.2) of Shapley.

Proposition (3.1). The game $(N, v)$ is convex.

Proof. Let $i$ be in $N$. We need to show that the inequality in equation (2) holds. Let $S \subseteq T \subseteq N \backslash\{i\}$. Then we need to show that

$$
\begin{aligned}
& {\left[3\left(\sum_{j \in T\{i\}} \sqrt{n_{j} \sigma_{j}^{2}}-\sqrt{\sum_{j \in T} n_{j i\}} \sigma_{j}^{2}}\right)-3\left(\sum_{j \in T} \sqrt{n_{j} \sigma_{j}^{2}}-\sqrt{\sum_{j \in T} n_{j} \sigma_{j}^{2}}\right)\right] \geq} \\
& {\left[3\left(\sum_{j \in S\{i\}} \sqrt{n_{j} \sigma_{j}^{2}}-\sqrt{\sum_{j \in S\{i\}} n_{j} \sigma_{j}^{2}}\right)-3\left(\sum_{j \in S} \sqrt{n_{j} \sigma_{j}^{2}}-\sqrt{\sum_{j \in S} n_{j} \sigma_{j}^{2}}\right)\right]}
\end{aligned}
$$

By removing terms that occur on both side and If we denote $\mathrm{A}=n_{i} \sigma_{i}^{2}, \mathrm{~B}=\sum_{j \in S} n_{j} \sigma_{j}^{2}$, and $\mathrm{C}=$
dividing both side by 3 , we reduce this to $\sqrt{\sum_{j \in T} n_{j} \sigma_{j}^{2}}-\sqrt{\sum_{j \in T} n_{j i\}} \sigma_{j}^{2}} \geq \sqrt{\sum_{j \in S} n_{j} \sigma_{j}^{2}}-\sqrt{\sum_{j \in S} n_{j i\}} \sigma_{j}^{2}} \quad \sum_{j \in T \backslash S} n_{j} \sigma_{j}^{2}$, Then we have to verify: 


$$
\sqrt{B+C}-\sqrt{A+B+C} \geq \sqrt{B}-\sqrt{A+B} \text { for } \mathrm{A} \geq 0, \mathrm{~B} \geq 0 \text { and } \mathrm{C} \geq 0 .
$$

this is equivalent to verifying the following inequality:

$$
\sqrt{B+C}+\sqrt{A+B} \geq \sqrt{B}+\sqrt{A+B+C} .
$$

$$
\text { Or } A+B+C+B+\sqrt{A+B} \sqrt{A+C} \geq A+B+C+B+\sqrt{A+B+C} \sqrt{B} \text {, }
$$

which holds for every non-negative $A, B$ and $C$ because

$$
(A+B)(B+C) \geq B(A+B+C) .
$$

By theorem (2.2) we can give an explicit formula for a stable split, namely the Shapley value of the game $(N, v)$. However, this might give too rosy picture and we want to point out the following observation: if there are two companies (i.e., $k=2$ ), then the Shapley value divides the money equally. This fact is not surprising since the game we defined does not differentiate between the two companies. In the succeeding sections, we look for ways to solve this phenomenon.

\section{The two companies case}

When we have two companies then the game we defined is as follows:

$$
\begin{aligned}
& v(\phi)=v(\{1\})=v(\{2\})=0 ; \\
& v(\{1,2\})=M .
\end{aligned}
$$

Thus, it is evident why there is no difference between the two companies. So, we look to incorporate additional information into the game that will reflect the differences. One such way would be to change the value of $v$ over the individual companies to be some loss factor for the companies (it is reasonable to assume that if the companies do not merge then they lose something). We offer to set

$$
v(\{i\})=3\left(\alpha_{i} \sigma-\sqrt{n_{i} \sigma_{i}^{2}}\right) .
$$

where $\sigma$ is the standard deviation of the merged companies, with $\alpha_{1}+\alpha_{2}=1 \quad$ and $v(\{1\})+v(\{2\})=-M$.

One reasonable way to choose the weights $\alpha_{i}$ is to set them to be proportional to the size of the companies.

Reviewing this case, we see that the attitude of the companies toward the money should be considered, and this leads us to thinking about utility functions.

\section{A Bernoulli game and Kahneman-Tverski utility function}

Daniel Bernoulli (1738) studied the game of tossing a fair coin till it turns tail. The player then gets $2^{i-1}$ cents where $i$ is the number of flips. How much money a player is willing to invest in this game? We calculate the game's expected value.

Let $X$ be a random variable for which $P\left(X=2^{i-1}\right)=\left(\frac{1}{2}\right)^{i}$, then its expected value is

$$
E(X)=1 \cdot \frac{1}{2}+2 \cdot\left(\frac{1}{2}\right)^{2}+2^{2} \cdot\left(\frac{1}{2}\right)^{3}+2^{3} \cdot\left(\frac{1}{2}\right)^{4}+\ldots=\frac{1}{2}+\frac{1}{2}+\frac{1}{2}+\ldots=\infty
$$

Hence, a player would be willing to invest any amount of money (an "infinite" amount) in the game. This is of course not reasonable. On the other hand, if the player estimates the value of its money using the function $u(x)=\sqrt{x}$ (which we call a utility function), then the expected value of the game is

$$
\begin{aligned}
& E u(X)=\sqrt{1} \cdot \frac{1}{2}+\sqrt{2} \cdot\left(\frac{1}{2}\right)^{2}+\sqrt{2}^{2} \cdot\left(\frac{1}{2}\right)^{3}+\sqrt{2}^{3} \cdot\left(\frac{1}{2}\right)^{4}+\ldots= \\
& =\frac{1}{2}\left(1+\frac{1}{\sqrt{2}}+\left(\frac{1}{\sqrt{2}}\right)^{2}+\left(\frac{1}{\sqrt{2}}\right)^{3}+\left(\frac{1}{\sqrt{2}}\right)^{4}+\ldots\right)=\frac{1 / 2}{1-\frac{1}{\sqrt{2}}}<\infty .
\end{aligned}
$$

So, this player will be willing to invest up to

$$
\left(\frac{1 / 2}{1-1 / \sqrt{2}}\right)^{2}
$$

in the game. In other words, the player would put in an amount that corresponds to the expected utility of the game.

Following this reasoning, we propose to use utility function in the solutions. In section 4 , we modified 
the game of section 3 to include information on losses of the two companies. Hence, we wish to choose utility functions that differentiate between losses and gains. Kahneman and Tverski develop a utility theory that puts losses in the center of attention. This is not done in the classical theory (i.e., expected utility theory in Kahneman-Tverski utility functions have the following distinctive characteristics:

1. They are defined regarding to a reference point $Z$.

2. They are concave above $Z$ and convex below.

3. The values of the derivative for values below $Z$ are greater than corresponding values above $Z$.

The point $Z$ is the turning point from losses to gains. The second item indicate that a player is indifferent for small changes, both for losses and gains. The last item says that people, for same amount of money, dislike losing more than they like earning Given the above, it is most natural to use these functions.

\section{Using utility function to split the emerging profit}

Next, we generalize the Shapley value to use utility function. Given a utility function $u(x)$, we emulate the process which calculates the Shapley value. First, we analyze this process. Suppose we are given a linear order $L$ on $N$, then $i$-th entry of $a^{L}$ is the marginal monetary contribution of company $i$ to the coalition $L(i)$. The Shapley value for company $i$ is the average of all its marginal contributions (over all orders). Define the vector $u\left(a^{L}\right)$ to be a real vector where its $i$-th entry is defined to be:

$$
u(v(L(i) \quad\{i\}))-u(v(L(i))) .
$$

That is, the marginal contribution of company $i$ in terms of utility. Using that we can define the following imputation:

$$
u(N, v)=\frac{v(N)}{k ! u(v(N))} \sum_{L} u\left(a^{L}\right),
$$

which gives each company a sum that is proportional to its average marginal contribution to the utility.

Using $\mathrm{u}(N, v)$ as a solution incorporates the tool of utility functions. However, this solution has a major disadvantage, since it assumes single utility function for all companies. We plan to lift this obstacle in succeeding article.

\section{Examples}

For the rest, we consider examples of merging some or all the following 3 companies.

Table 1. Examples of merging

\begin{tabular}{|c|c|c|c|}
\hline Company & Members & Net Premium & $\begin{array}{c}\text { Standard } \\
\text { Deviation }\end{array}$ \\
\hline 1 & 1000 & 150 & 316.23 \\
\hline 2 & 10,000 & 100 & 500 \\
\hline 3 & 40,000 & 70 & 600 \\
\hline
\end{tabular}

The emerging profit in case companies 1 and 2 merge is $27,029.41$, and the emerging profit if all 3 companies merge is $148,847.85$.

Example 1: The split under the Shapley value method in case all companies merge can be calculated by using Matlab or a similar tool to obtain:

$$
\varphi_{1}=18,912.88, \varphi_{2}=64,536.8, \varphi_{3}=65,398.17
$$

Example 2: The split of the emerging profit in case companies 1 and 2 merge is:

a) under the Shapley value method, the split follows applying section 4 with :

$$
\alpha_{1}=\frac{n_{1}}{n_{1}+n_{2}}, \quad \alpha_{2}=\frac{n_{2}}{n_{1}+n_{2}}
$$

The split is for the first company $10,935.83$ and for the second 16,093.58.

b) companies, and using the following KahnemanTverski utility function:

$u(x)=\left\{\begin{array}{c}\ln (1+x) \text { if } x \geq 0 \\ 2 \ln (1-x) \text { if } x<0\end{array}\right.$.

The split is for the first company $12,491.38$ and for the second 14,538.03.

We notice that the splitting of the profit depends on the utility functions used.

\section{Conclusion}

We intended to study the emerging profit in case of insured groups that merge into a single group. We prove in introduction section that there emerges a profit due to a reduced risk when groups merge to a single group. Shapley value method discussed in section 2 or using a given utility functions for all groups as discussed in section 5 may be used to derive a splitting of the profit between the groups' and we observe in section 6 that the result may depend upon the method chosen to calculate the splitting.

In section 2, we see that the Shapley value method in the case of two companies leads to an equal split of the profit. This is not the case in general. 
We suggest in section 3 a modified game where information on losses of the two companies is considere. One may use utility functions as developed in Kahneman, D., \& Tversky, A. (1979)

that emphasizes losses that differs from the classical Expected Utility Theory by Neumann, J. von, \& Morgenstern, O. (1944).
We will generalize the case of a given utility functions for all groups to generalized to the case where each group uses its own utility function in a following article.

We observe in section 6 that to calculate the splitting of the profit one may need Matlab or a similar tool.

\section{References}

1. Bernoulli, D. (1954). Specimen Theoriae Novae de Mensura Sortis. Commentarii Academiae Scientiarum Imperialis Petropolitanae, V (1738), 175-192 (English translation: Exposition of a new theory on the measurement of risk. Econometrica, 22 (1954), 23-36).

2. Dehling, H. G. (1998). Daniel Bernoulli and the St. Petersbourg Paradox. Nieuw Archief Voo rWiskunde, Vierdeserie, 15, 223-227.

3. Shapley, L. S. (1929). Cores of Convex Games. International Journal of Game Theory, 1, 11-26.

4. Weber, R.J. (1994). Games in Coalitional Form. Hand Book of Game Theory, 2, 2285-1303.

5. Kahneman, D., \& Tversky, A. (1979). Prospect Theory: An Analysis of Decision Under Risk. Econometrica, 47(2), 263-291.

6. Neumann, J. von, \& Morgenstern, O. (1944). The Theory of Games and Economic Behavior. Princeton University Press.

7. Borch, K. (1974). The Mathematical Theory of Insurance. D.C. Heath and Company. 